\title{
Surgical Management with Self-Locking Pins and Circumferential Wiring for Treating Comminuted Patella Fractures Achieved Seiza-Style Sitting
}

\author{
Kazuha Kizaki $^{1}$ Soshi Uchida ${ }^{2}$ Noboru Funakoshi ${ }^{1}$ \\ Fumiharu Yamashita ${ }^{1}$ \\ ${ }^{1}$ Department of Orthopaedic Surgery and Rheumatology, Kyoto \\ Shimogamo Hospital, Kyoto, Japan \\ ${ }^{2}$ Department of Orthopaedic Surgery and Sports Medicine, \\ Wakamatsu Hospital of the University of Occupational and \\ Environmental Health, Fukuoka, Japan \\ ${ }^{3}$ Division of Physical Therapy, Kyoto Shimogamo Hospital, Kyoto, Japan
}

\author{
Daisuke Mori ${ }^{1}$ \\ Ryosuke Nakai ${ }^{3}$
}

Joints 2019;7:218-221.

\begin{abstract}
Address for correspondence Kazuha Kizaki, MD, Department of Orthopaedic Surgery and Rheumatology, Kyoto Shimogamo Hospital, 17 Shimogamo, Sakyo-ku Ward, Kyoto Prefecture, Kyoto 606-0866, Japan (e-mail: kazuhakizaki@gmail.com).
\end{abstract}

\author{
Abstract \\ Keywords \\ - patella fracture \\ - comminuted \\ - surgical management
}

Surgical fixation of comminuted patella fractures is technically challenging. Despite the fact that tension band wiring or screw fixation are the most common surgical procedures, these surgical techniques have crucial limitations for laterally scattered fragments. We demonstrate two cases with comminuted patella fractures undergoing surgical fixation with self-locking pin and circumferential wiring, confirming the rigid fixation achieving deep knee flexion. After midline longitudinal skin incision, $2.0 \mathrm{~mm}$ stainless pins (AiMedic MMT, Tokyo, Japan) were inserted radially to penetrate each fragment and $1.2 \mathrm{~mm}$ stainless wire was circumferentially looped and self-locked in the holes on the pins, which satisfied rigid fixation. Any restrictions in range of motion and weight bearing were required postoperatively and 3- to 6-month postoperative cares achieved seiza-style sitting with deep knee flexion.

\section{Introduction}

Patella fractures are common fractures, comprising approximately $1 \%$ of all fractures. ${ }^{1}$ Surgical intervention is recommended when the articular incongruity (step-off) is more than $2 \mathrm{~mm}$, the displacement of the fragments is more than $3 \mathrm{~mm}$, or the extensor mechanism is disrupted. ${ }^{2}$ The tension band wiring (TBW) and screw fixation techniques are the most standard surgical options for patella fractures, ${ }^{3}$ but these techniques are not ideal theoretically for comminuted patella fractures because fragments are scattered radially. Maruo et al have demonstrated the surgical technique using self-locking pins and circumferential wiring for patella fractures $^{4}$ with $2.0 \mathrm{~mm}$ stainless self-locking pins (Aimedic MMT, Tokyo, Japan) and approximately 40,000 self-locking

received

May 28, 2018

accepted after revision

April 18, 2021

published online

June 18, 2021 pins have been used in our country in the last 3 years. In spite of the widespread utilization of this surgical technique in Japan, it has not been widespread outside of our country, because there is a lack of English literature demonstrating this surgical technique. In this case report, the surgical technique with self-locking pins and circumferential wiring was performed, which made it endurable to position deep knee flexion. For Judo, Kendo, and Sadō, deep flexion of the knee like seiza-style sitting is essential ${ }^{5}$ and patients' satisfaction after surgery is truly dependent on whether they can pose seiza-style sitting postoperatively. We also proposed the suitable postoperative rehabilitation protocol following this surgical technique. The purpose of this case report is to demonstrate the rigid fixation of self-locking pins and circumferential wiring for treating comminuted patella
DOI https://doi.org/ C 2021. The Author(s)

$10.1055 / \mathrm{s}-0041-1730379$.

ISSN 2282-4324.

This is an open access article published by Thieme under the terms of the Creative Commons Attribution-NonDerivative-NonCommercial-License, permitting copying and reproduction so long as the original work is given appropriate credit. Contents may not be used for commercial purposes, or adapted, remixed, transformed or built upon. (https://creativecommons.org/ licenses/by-nc-nd/4.0/) Georg Thieme Verlag KG, Rüdigerstraße 14, 70469 Stuttgart, Germany 
fractures, and achieving a seiza-style sitting position after 3- to 6-month postoperative therapy sessions.

\section{Surgical Technique}

In a supine position on a table under general anesthesia, a tourniquet is used. After a longitudinal midline skin incision is made, the extensor retinaculum is exposed. Fracture fragments are fixed with forceps outside of the extensor retinaculum and $2.0 \mathrm{~mm}$ stainless pins (Aimedic MMT) are radially inserted from the outside of the extensor retinaculum, penetrating each fragment to unite them into one block. Here, the extensor retinaculum should not be peeled off from the fragments to retain blood supply. Next, $1.2 \mathrm{~mm}$ stainless cable wire (Aimedic MMT) is circumferentially looped around the patella, passing through oval crimps on top of the inserted stainless pins which have two holes on the top each. Here, it is not necessarily required to insert the pins too much to directly attach fragments, since soft tissues including the extensor retinaculum cover fragments to unite into one piece when pins are inserted. After circulating the patella, the stainless cable wire is pulled using a cable tensioner with appropriate tension depending on the fracture type and quality of bone. While keeping rigid fragments fixation with the appropriate tension on the cable, the oval crimp on top of each pin is pinched using the cable pin crimper and, therefore, the stainless pin and cable wire are rigidly self-locked in the oval cramp (that is why, this surgical technique is named as self-locking pins and circumferential wiring). Finally, the free cable wire is cut. The key concept of this surgical technique is anatomical reduction using forceps, and rigid fixation with stainless pins in combination with the circumferential cable wire.

\section{Case Presentation}

\section{Case 1}

A 71-year-old female patient who fell with her right knee hitting the ground was presented to us and was diagnosed with a comminuted displaced patella fracture classified into AO type 34-C3 by plain radiographs (-Fig. 1) and surgical treatment was adapted with self-locking pins and circumferential wiring. The surgery time was 120 minutes. Postoperative plain radiographs are shown in -Fig. 1. Postoperatively, weight bearing was initiated with extensor knee brace at day 1 after surgery. Range of motion (ROM) was restricted at 90 degrees until postoperative day (POD) 14. After POD 14, extensor knee brace was removed and unrestricted knee bending exercises were started.
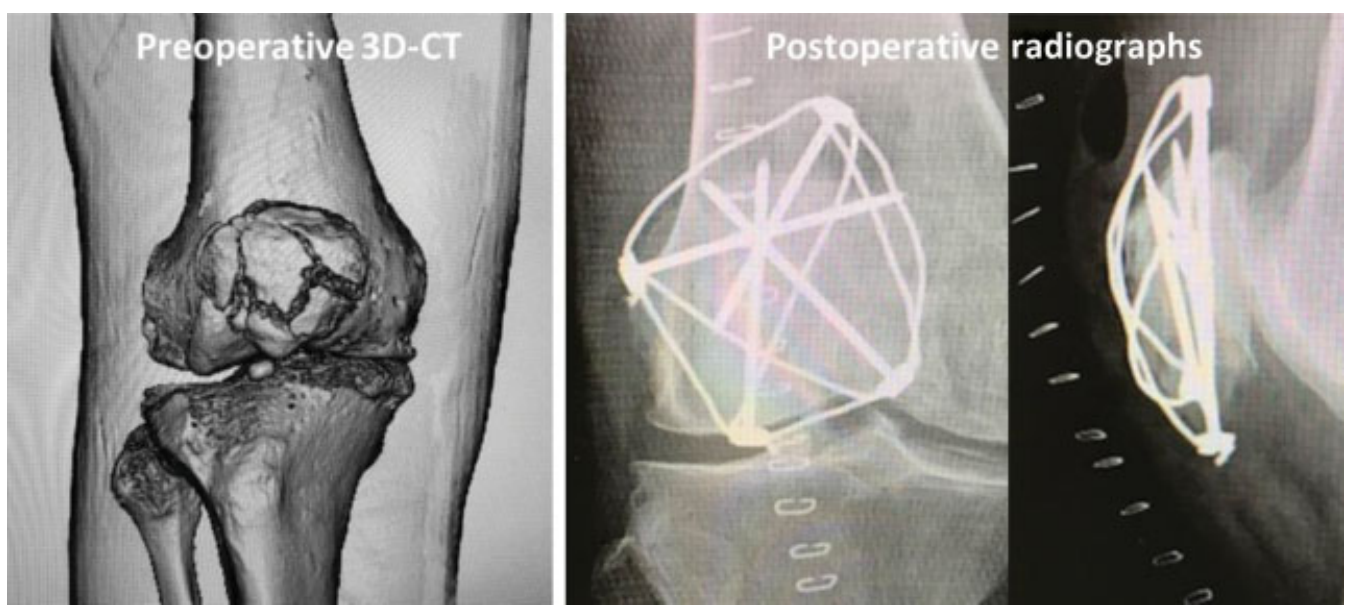

Fig. 1 Preoperative three-dimensional computed tomography (3D-CT) and postoperative radiographs in case 1.

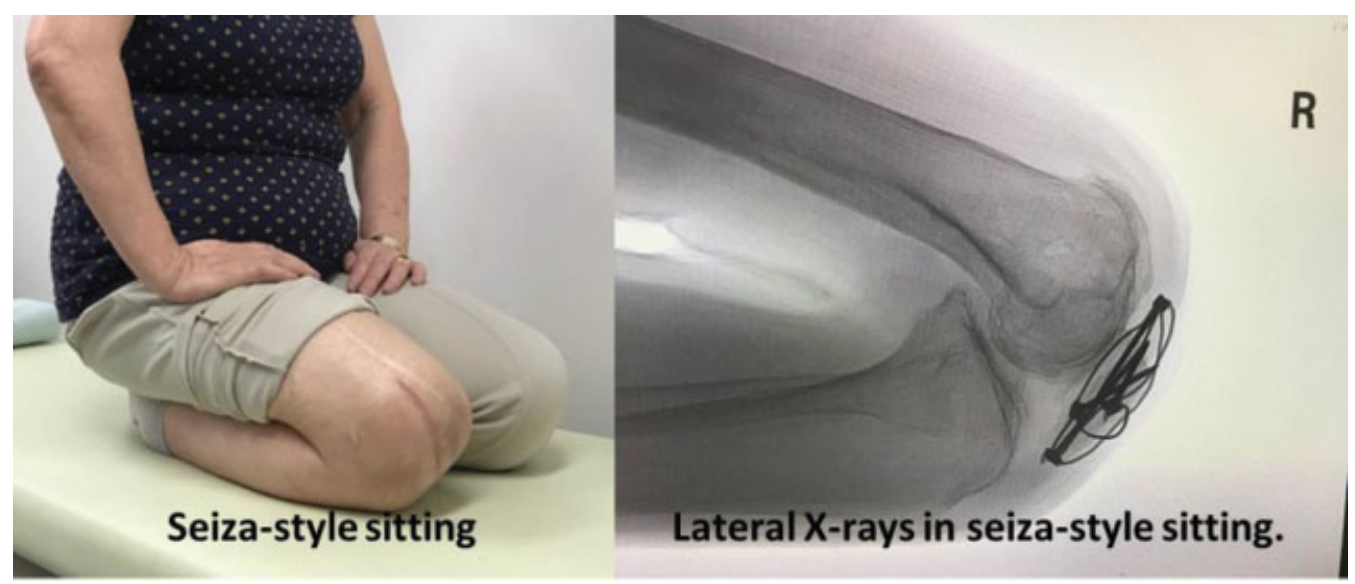

Fig. 2 Seiza-style sitting at 6-month postoperative follow-up in case 1. 
She had a slight knee contracture postoperatively and physical therapy was continued for 6 months. Finally, her knee contracture was completely restored and she was able to achieve seiza-style sitting with deep knee flexion, as shown in - Fig. 2. She complained of anterior knee pain when bending her knee at a 6-month postoperative followup visit, though she did not request removing the hardware implants.

\section{Case 2}

A 67-year-old male patient who hit his left knee on the ground while he was cycling and was diagnosed with a comminuted patella fracture classified into AO type 34-C3 (-Fig.3). As his extensor mechanism was disrupted, surgical intervention was selected using the described surgical technique. After a midline longitudinal skin incision, two 2.0-mm Kirschner wires (K-wires) were first inserted to fix the disrupted inferior pole fragments. Then, radial pinning was inserted into each fragment and circumferential wiring was conducted as shown in -Figs. 3 and $\mathbf{4}$. The surgery time was 150 minutes. Postoperatively, weight bearing was initiated with a knee brace as was tolerable and any ROM restriction was set. At 1 week after surgery, he was able to walk with crutches. At the 4-week postoperative follow-up visit, the flexion and extension of the knee were 135 degrees and -15 degrees and he walked without crutches. At the 3month postoperative visit, he demonstrated seiza-style sitting with deep knee flexion as shown in - Fig. 5. He also complained of recurrent anterior knee pain when bending his knee, though his complaint was not severe enough to require removing the hardware implants.

\section{Discussion}

The most important finding of this case report is the excellent rigidity of self-locking pins and circumferential wiring surgical procedures. This article described two cases with comminuted patella fractures undergoing internal fixation with self-locking pins and circumferential wiring. In case 1 , we demonstrated that this surgical technique could allow rigid fixation to endure maximum flexion of the knee during surgery, and the patient could accomplish posing seiza-style sitting at 6 months after surgery. In case 2, we revealed the rapid postoperative rehabilitation enabled walking within 4-week postoperative care and positioning seiza-style sitting at 3-month postoperative care.
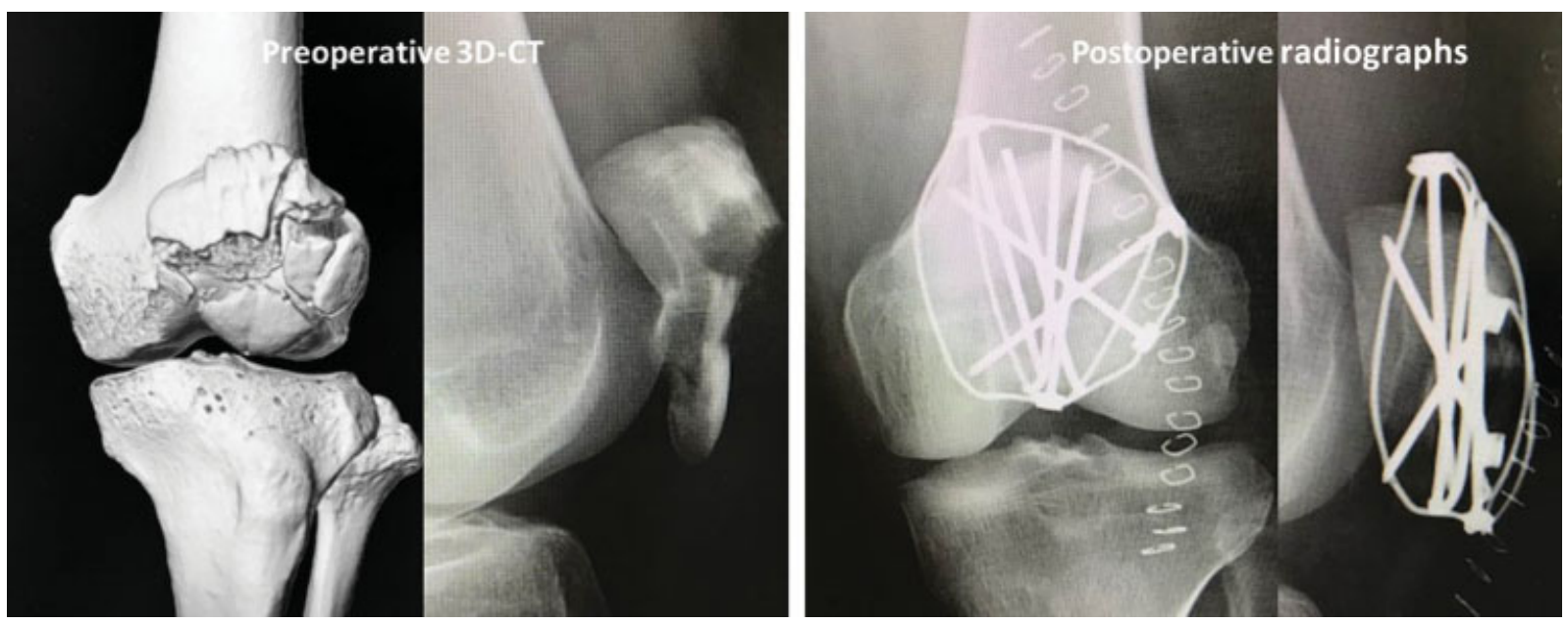

Fig. 3 Preoperative three-dimensional computed tomography (3D-CT) and postoperative X-rays in case 2.
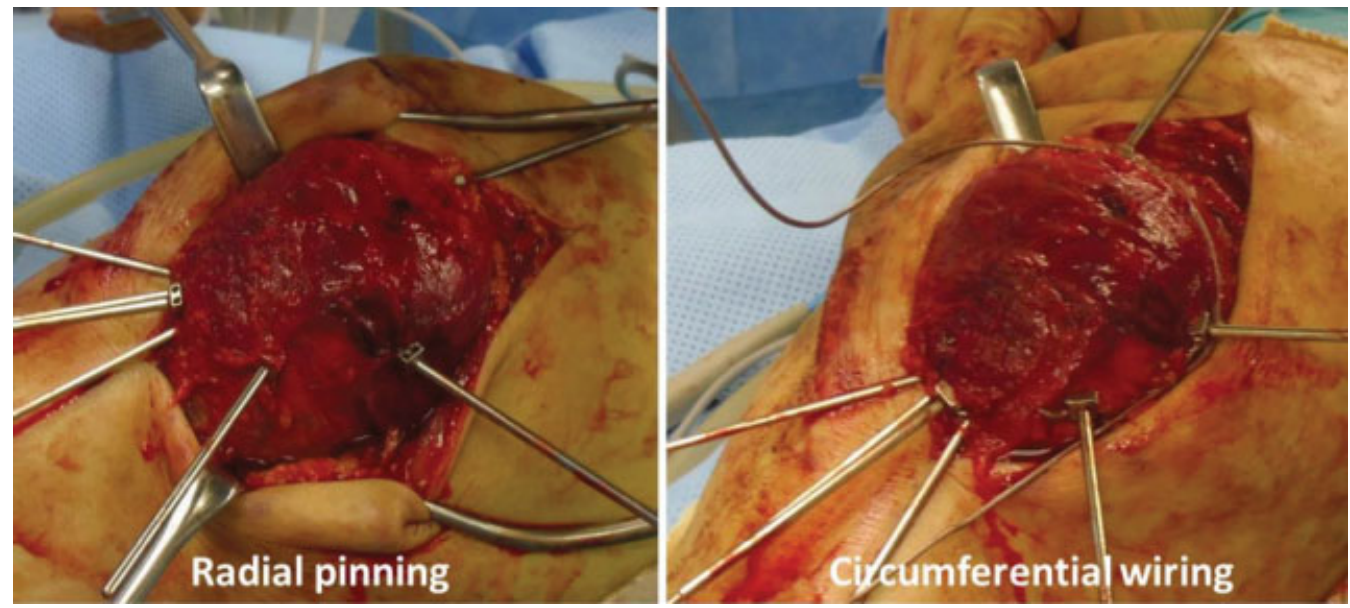

Fig. 4 Surgical view with self-locking pin and circumferential wiring in case 2. 

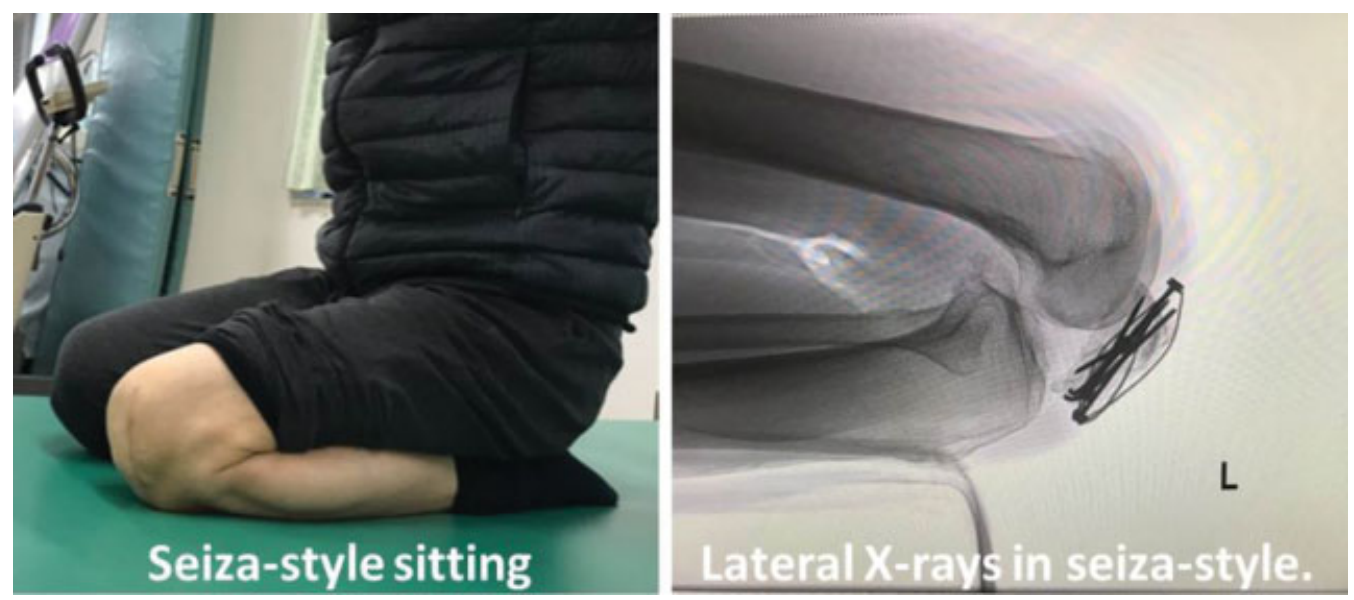

Fig. 5 Seiza-style sitting at 3-month postoperative follow-up in case 2.

Comminuted patella fractures are technically demanding. ${ }^{6}$ TBW and screw fixation have been recognized as standard procedures, ${ }^{7}$ but these surgical options have limitations for treating comminuted patella fractures with detachment of fragments radially. Fear of detachment or nonunion, restrictions of ROM, and weight bearing are sometimes increased. Prolonged restriction often causes knee contracture and quadriceps muscle atrophy. ${ }^{8}$ This surgical technique enables rigid fixation of a comminuted patella fracture to allow early rehabilitation after surgery.

A potential complication of K-wire/pin fixation is migration of the inserted K-wire/pin. Previously, self-locking $\mathrm{K}$-wires, in which the ends were bent to form a loop, and a cerclage wire was passed through them, showed successful outcomes to avoid migration of the K-wires. ${ }^{9}$ This surgical technique also avoids migration of the pins owing to the selflocking pin system fixed with the circumferential wire. Also, migration of the cerclage wire is a common problem in TBW.To avoid the wire and pin migration, ring pins are used as the wire passes through the ring pins, and they prevent migration with each other. ${ }^{10,11}$ The self-locking pins in this surgical technique also prevent wire and pin migration, as the circumferential cable wire is locked in the oval crimps on top of the pins.

However, this surgical technique has several disadvantages. First, this surgical technique has not yet been fully certified in biomechanical studies. Further biomechanical studies are required to elucidate the biomechanical benefit of this surgical technique. Second, in both clinical cases, surgery time was longer than conventional surgical techniques for patella fractures (e.g., TBW and screw fixation). Also, this surgical technique increased expenses. For case 1 and 2, it cost approximately 5,000 US dollars for the implants. From an economic point of view, this technique may be limited only for comminuted patella fractures classified into AO type $34-\mathrm{C} 3$, requiring rigid fixation.

\section{Conclusion}

We showed the rigid fixation of self-locking pin and circumferential wiring for comminuted patella fractures, achieving seiza-style sitting and proposed the suitable postoperative rehabilitation protocol without any ROM nor weight-bearing restriction. However, this surgical technique has disadvantages: postoperative hardware-related irritation and high implant cost. This surgical technique may be restricted only for comminuted patella fractures classified into AO type 34-C3.

\section{Conflict of Interest \\ None declared.}

\section{Acknowledgment}

We truly appreciate Gary B. Perry for proofing assistance in English.

\section{References}

1 Scolaro J, Bernstein J, Ahn J. Patellar fractures. Clin Orthop Relat Res 2011;469(04):1213-1215

2 Schuett DJ, Hake ME, Mauffrey C, Hammerberg EM, Stahel PF, Hak DJ. Current treatment strategies for patella fractures. Orthopedics 2015;38(06):377-384

3 Yoon YC, Sim JA, Hong JH. Surgery of patellar fractures using a medial parapatellar approach. J Orthop Surg (Hong Kong) 2017;25 (02):2309499017719378

4 Maruo A, Fujita H, Oka S, et al. Clinical results of self-locking pin and circumferential wiring, "Himawari Method" for patellar fractures. Fracture 2009;31(03):644-648

5 Demura S, Uchiyama M. Effect of Japanese sitting style (seiza) on the center of foot pressure after standing.J Physiol Anthropol Appl Human Sci 2005;24(02):167-173

6 Böstman O, Kiviluoto O, Nirhamo J. Comminuted displaced fractures of the patella. Injury 1981;13(03):196-202

7 Gwinner C, Märdian S, Schwabe P, Schaser KD, Krapohl BD, Jung TM. Current concepts review: fractures of the patella. GMS Interdiscip Plast Reconstr Surg DGPW 2016;5(05):Doc01

8 Lazaro LE, Wellman DS, Sauro G, et al. Outcomes after operative fixation of complete articular patellar fractures: assessment of functional impairment. J Bone Joint Surg Am 2013;95(14):e96

9 Us AK, Kinik H. Self locking tension band technique in transverse patellar fractures. Int Orthop 1996;20(06):357-358

$10 \mathrm{Kim}$ MB, Lee YH, Shin WC, Baek GH. Locked tension band wiring using ring pins for patellar fractures: a method of minimising implant migration. Arch Orthop Trauma Surg 2014;134(11):1537-1543

11 Kyung MG, Lee SH, Kim MB. Complications related to implant fixation of patellar fractures: comparison of ring pins versus bent K-wires. J Knee Surg 2017;30(06):560-564 